\title{
Integrate-and-fire models of insolation-driven entrainment of broadcast spawning in corals
}

\author{
Bharath Ananthasubramaniam • Roger M. Nisbet • \\ Daniel E. Morse · Francis J. Doyle III
}

Received: 20 November 2009 / Accepted: 8 March 2010 / Published online: 13 April 2010

(C) The Author(s) 2009. This article is published with open access at Springerlink.com

\begin{abstract}
The circa-annual cycle of gametogenesis produces mature gametes at the spawning "season" for successful mass spawning of broadcast corals. We develop a bioenergetic integrate-and-fire model that reveals how annual insolation rhythms can entrain the gametogenetic cycles in tropical hermatypic corals to the appropriate spawning season, since photosynthate is their primary source of energy. In the presence of short-term fluctuations in the energy input, a feedback regulatory mechanism is likely required to achieve coherence of spawning times to within one lunar cycle, in order for subsequent signals such as lunar and diurnal light cycles to unambiguously determine the "correct" night of spawning. The feedback mechanism can also provide robustness against population heterogeneity that may arise due to genetic and environmental effects. We
\end{abstract}

B. Ananthasubramaniam

Institute of Collaborative Biotechnologies,

University of California, Santa Barbara,

Santa Barbara, CA 93106-5080, USA

R. M. Nisbet

Ecology, Evolution and Marine Biology,

University of California, Santa Barbara,

Santa Barbara, CA 93106-9620, USA

D. E. Morse

Molecular, Cellular and Developmental Biology,

University of California, Santa Barbara,

Santa Barbara, CA 93106-9610, USA

F. J. Doyle III ( $\varangle)$

Chemical Engineering, University of California,

Santa Barbara, Santa Barbara, CA 93106-5080, USA

e-mail: frank.doyle@icb.ucsb.edu solve the integrate-and-fire bioenergetic model numerically using the Fokker-Planck equation and use analytical tools such as rotation number to study entrainment.

Keywords Bioenergetic integrate-and-fire model • Broadcast spawning • Entrainment • Insolation • Coral reproduction $\cdot$ Biological rhythms . Computational biology

\section{Introduction}

A large number of sessile and sedentary marine invertebrates (Giese 1959), including corals, gastropods, ascidians, bivalves (Babcock et al. 1992), to name a few, reproduce by broadcast spawning, i.e., by releasing their gametes-spermatocytes and oocytes-into the water column, where gametes from different individuals fertilize to produce larvae. Synchronous release of these gametes vitally determines and maximizes reproductive success and maintenance of genetic diversity in the population (Crean and Marshall 2008). One instance of extremely tight synchrony in spawning is observed in a subset of corals known as broadcast spawning corals.

Broadcast spawning corals reproduce in synchronized mass spawning events (Babcock et al. 1986) that most typically occur annually close to the period of peak insolation in a window of a few hours after sunset during the waning moon phase close to the night of the full moon. It is postulated that these corals have perfected the control of initiation of gamete development (gametogenesis) for spawning, since gametes have to be at the optimal stage of development for 
successful fertilization after release. These corals appear to have a hierarchical perception of time to facilitate synchronized spawning: circa-annual (of the order of months), circa-lunar (of the order of days), and circadiel (of the order of hours) scales of timekeeping that we now outline based on the probable underlying coral physiology (similar observations have been made in Babcock et al. (1986) and van Woesik et al. (2006)).

The gametogenetic cycle spans typically 3-9 months, with oogenesis having a longer development time than spermatogenesis (Wallace 1985). Therefore, oogenesis and spermatogenesis are appropriately initiated to ensure that both gametes are concurrently mature at the time of spawning. Gametogenesis thus occurs in the circa-annual scale to produce gametes of sufficient maturity at the time of spawning. Solar insolation and sea surface temperature (SST) have been proposed as possible exogenous environmental inputs for scheduling reproductive processes in the circa-annual scale (Baird et al. 2009). Although most spawns occur at SST between $28^{\circ} \mathrm{C}$ and $30^{\circ} \mathrm{C}$ (close to the peak SST in the respective region), SST is unable to explain the differences in spawning times observed between the east and west coast of Australia with similar SST patterns (Baird et al. 2009). On the other hand, changes in solar insolation have been empirically shown to be good predictors of spawning times of Montastraea annularis in the Caribbean by van Woesik et al. (2006). The signals that drive synchrony in circa-annual time scales are often referred to as "ultimate" triggers whereas the drivers in the circa-lunar and circa-diel scales are termed 'proximal' triggers.

Spawning in several coral species occurs sometime between the full moon and several days after the full moon, depending on the species and geographical location. Nevertheless, spawning is consistent from year to year at the same location for the same species (Table 1, Mendes and Woodley 2002). This points to the presence of a circa-lunar (or intralunar cycle) tracking of time by corals, although the exact mechanism relating the time of spawning to the lunar cycle remains unknown. The understanding of this causation by lunar irradiance is complicated by the presence of secondary effects of the lunar cycle such as tides and length of dark period, whose effects cannot be decoupled.

Finally, most observed coral spawnings have been after dark or twilight, and the spawning event is completed in a window of approximately $1 \mathrm{~h}$. The times of spawning within the day have also been remarkably consistent at a given location for a particular species. At the circa-diel level, the hour of spawning has been hypothesized to be "triggered" by diurnal light cycles and time of spawning has been successfully manipulated by altering the light conditions (Knowlton et al. 1997; Brady et al. 2009). There is also some evidence of synchrony between coral colonies being possibly driven by chemical exchanges of hormones through diffusion in the water column (Atkinson and Atkinson 1992).

Although considerable research has been directed toward understanding the factors that contribute to the robust synchrony in spawning, relatively little is known about the pathways involved in the "triggers", a problem that is exacerbated by the incomplete knowledge of coral physiology. In this paper, we take an analytical approach to elucidating mechanisms that permit entrainment to environmental cycles with minimal assumptions regarding coral physiology. We address the questions: Can a single environmental driver such as insolation with annual periodicity in conjunction with simple bioenergetic mechanisms robustly (in the presence of noise) entrain a coral to an circa-annual cycle to limit the possible spawning to about 1 month, whereupon the drivers acting on the finer circa-lunar and circa-diel time scales can trigger spawning at the 'correct' time? If this is possible, what are the evolutionarily selected, biologically consistent mechanisms that can achieve this and how narrow a window can spawning be restricted to?

Tropical hermatypic corals engage in a symbiotic relationship with Symbiodinium (micro-algae) that live inside the coral tissue. There is sufficient evidence (Muscatine et al. 1984; Edmunds and Davies 1986) to conclude that hermatypic corals derive most of their energy (up to $80 \%$ ) from the Symbiodinium, especially in shallow reef corals in well-lit tropical waters (as mentioned earlier, empirical evidence of this correlation was presented in van Woesik et al. (2006)). Symbiodinium produce energy-rich compounds (photosynthate) from photosynthesis and to a first approximation, the photosynthate produced by the Symbiodinium is proportional to the amount of insolation incident on the coral. This photosynthate fufills the high energy demands of reproduction (Rinkevich 1989) allowing us to use insolation as a key driver determining the corals' capability to spawn. This hypothesis is also reinforced by the observation that spawning in many species occurs during periods of peak insolation (Penland et al. 2004).

Although our results are equally applicable to other broadcast spawning organisms in response to environmental forcing, we present our findings using the entrainment of coral spawning to exogenous insolation as an example. In this work, we do not consider the evolution of synchrony from interactions between coral 
individuals, as suggested in Atkinson and Atkinson (1992). We also defer to a separate treatment the effect of other exogenous signals, such as lunar phase, lunar irradiance, and diurnal light cycles on the spawning time at the circa-lunar and circa-diel scales.

We use a bioenergetic model of the coral, in which some part of the energy derived from photosynthesis by the endosymbiotic Symbiodinium is allocated to the reproductive reserves. We use the accumulated reserves as an index of the reproductive state of the coral. When sufficient reserves have been accumulated, spawning can occur. In this regard, we develop a simple "integrate-and-fire" (I\&F) model for corals that is mathematically similar to models for neurons (Knight 1972). We regard this model as an extreme simplification of the dynamic energy budget theory-based models, such as the one recently proposed for coral symbiosis by Muller et al. (2009). Although a great deal is known about coral physiology, the interactions between the host, the symbiont, and the environment are highly complex, making more detailed mechanistic models uninstructive. Finally, we find these low dimension I\&F models extremely attractive for ultimately studying populations of interacting coral individuals.

The entrainment mechanisms we study, where individuals achieve coherence with an external periodic driver, is complementary to the mechanisms of synchrony where coherence is achieved through interaction (or coupling) between individuals; henceforth, we use the terms "entrainment" and "synchrony" to refer specifically to these two complementary mechanisms. A combination of entrainment and synchrony is used in many biological systems to produce robust rhythms, such as the mammalian circadian system (To et al. 2007).

Our bioenergetic entrainment model of synchronized reproduction shares many similarities with the globally coupled map models of synchronized "masting" or seeding in trees (Satake and Iwasa 2000, 2002). Analogous to our model, the map of masting proposed by Satake and Iwasa $(2000,2002)$ also tracks the reproductive reserves of individuals supplied by energy from photosynthesis, and masting occurs when the reserves reach a threshold. Using a globally coupled map, Satake and Iwasa $(2000,2002)$ capture the presence or absence of masting in a year and the correlation in the number of seeds produced in a year by different masting individuals. Thus, this model of the number of seeds produced by an individual tree from year-to-year (discrete-time) coupled by pollen exchange explores a mechanism of "synchrony" rather than a mechanism of "entrainment".
In contrast, we are interested in the coherence in spawning times within a year produced by a seasonal energy input with fluctuations through an entrainment mechanism without interaction between individuals (uncoupled) and without regard for the number of gametes released in a spawn. Moreover, individuals in a population receive the same mean insolation input with fluctuations uncorrelated between individuals. Satake and Iwasa (2000) further show that, under the conditions imposed on our model, individual trees in their model only exhibit chaotic masting.

\section{Bioenergetic entrainment model}

We consider a population of $N$ noninteracting coral individuals. Each coral individual receives photosynthate produced by the Symbiodinium from the incident solar insolation at an average rate $S_{t}$. The average incident insolation and, hence, the average photosynthate production rate are assumed to be the periodic entrainment driver with a period of 1 year, i.e., $S_{t+1}=$ $S_{t}$. The maximum insolation at a given geographical location is dependent on the latitude and time of the year. The maximum insolation profile has two peaks between the tropics (on the days when the sun is exactly overhead), while it has only one peak outside the tropics. Nevertheless, for simplicity, we consider a sinusoid with seasonal variation $\alpha$ and a period of 1 year as the mean energy input (idealized insolation in energy equivalents):

$S_{t}=1+\alpha \sin (2 \pi t-\phi), 0<\alpha<1$.

We present examples in the Discussion of our model with the theoretical maximum insolation profile at specific geographic locations. Moreover, the incident insolation is fluctuating constantly with changes in atmospheric conditions such as cloud cover and particulate matter in the air and water. These short-term fluctuations are modeled as additive Gaussian noise $\eta_{t}$ in the energy input with a coefficient of variation $(\mathrm{CV})$ with respect to the mean energy input $S_{t}$.

The individual's progress toward spawning at time $t$ is characterized by the quantity of its reproductive reserves, $X_{t}$. A fraction $\lambda$ of the photosynthate received by each coral is allocated to reproduction (see Fig. 1). This allocation fraction $\lambda$ might dependent on the quantity of reproductive reserves $X_{t}$ and time $t$, i.e., $\lambda=\lambda\left(X_{t}, t\right)$, and $0 \leq \lambda \leq 1$. Each possible choice of allocation $\lambda$ can be considered one "strategy" to achieve entrainment. We further assume that all corals are identical and employ the same strategy. Since our 


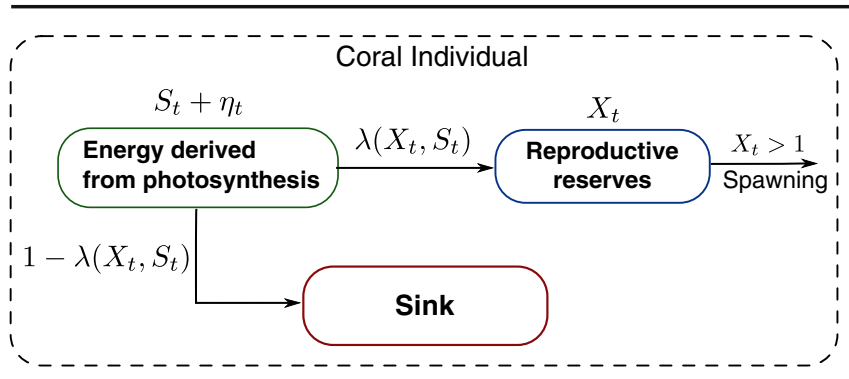

Fig. 1 Allocation to the reproductive reserves $X_{t}$ of energy obtained by a coral individual from its photoautotropic symbiont derived from insolation $S_{t}$ with fluctuations $\eta_{t}$ with the remaining energy going toward growth, somatic maintenance, and other reserves. $\lambda\left(X_{t}, S_{t}\right)$ represents the fractional allocation toward reproduction

premise is that the coral has no intrinsic timekeeper and thus has no "sense" of time, we restrict our study to strategies where the allocation only depends on the quantity of reserves and the mean energy input rate, i.e., $\lambda=\lambda\left(X_{t}, S_{t}\right)$.

The energy allocated to reproduction might not lead to an equal increase in the reserve level due to maintenance costs. ${ }^{1}$ Here we consider a maintenance cost $f\left(X_{t}, t\right)$ that is linearly dependent on the quantity of reserves $X_{t}$ and can vary with the amount of solar insolation. Thus, the maintenance cost is of the form $f\left(X_{t}, t\right)=\gamma m(t) x$, where $\gamma$ is the maintenance rate constant and $m(t)$ is the time dependence of the maintenance cost.

A coral spawns when its reserve $X_{t}$ reaches the normalized threshold of 1 and its reproductive reserves are expelled as gametes ( $X_{t}$ is reset to 0 ). $X_{t}$ is the total energy invested in the reproductive apparatus, such as gonads and gametes, by the coral; we represent all quantities in our model in energy equivalents. $N(x, t)$ is the number of coral individuals with reproductive reserves $X_{t}=x$ at time $t$; since the corals are identical and noninteracting, $N(x, t)$ and its dynamics are sufficient to represent the spawning behavior of the population. At any given time, since there is no coral birth or death, the total number of corals in the system is constant, $\int_{0}^{1} N(\xi, t) d \xi=N$. With a sufficiently large coral population, we can work equivalently with the fraction of individuals $P(x, t)=\frac{N(x, t)}{N}$ with reserves $X_{t}=x$ at time $t$. The dynamics of the fraction of individuals at each state at time $t$ is characterized by the following partial differential equation (PDE) (the development of this Fokker-Planck PDE from a stochastic energy

\footnotetext{
${ }^{1}$ The term maintenance has many specific definitions especially in dynamic energy budget theory (Kooijman 2000). Here, we use it to represent energy costs to sustain a certain quantity of reproductive reserves.
}

allocation model for the coral is presented in the Appendix A):

$$
\begin{aligned}
\frac{\partial P(x, t)}{\partial t}= & -\frac{\partial}{\partial x}\left[\left(-\gamma f(t) x+\frac{\lambda\left(x, S_{t}\right)}{T_{0}} S_{t}\right) P(x, t)\right] \\
& +\frac{\mathrm{CV}^{2}}{T_{0}^{2}} \frac{\partial^{2}}{\partial x^{2}}\left[\lambda^{2}\left(x, S_{t}\right) P(x, t)\right]
\end{aligned}
$$

where $T_{0}$ is the interval between successive spawning events for an individual coral under constant energy input $\alpha=0$, no fluctuations $\eta_{t}=0$, and no maintenance $\operatorname{costs} \gamma=0$. The spawning event followed by the reset of the reserve to 0 is imposed on Eq. 1 with boundary conditions

\section{$P(1, t)=0: \quad$ spawning when $x=1$}

and

$$
\begin{gathered}
\lambda\left(0, S_{t}\right) P(0, t)-\left.\frac{\mathrm{CV}^{2}}{T_{0}^{2}} \frac{\partial}{\partial x} \lambda^{2}\left(x, S_{t}\right) P(x, t)\right|_{x=0} \\
=-\frac{\mathrm{CV}^{2}}{T_{0}^{2}} \frac{\partial}{\partial x} \lambda^{2}\left(x, S_{t}\right) P(x, t) \mid \begin{array}{l}
\text { individuals that } \\
\text { spawned are added } \\
\text { to individuals at } \\
x=0 .
\end{array}
\end{gathered}
$$

We are interested in the fraction of individuals that spawn at any time $t$, which is

$F(t)=-\left.\frac{\mathrm{CV}^{2}}{T_{0}^{2}} \frac{\partial}{\partial x} \lambda^{2}\left(x, S_{t}\right) P(x, t)\right|_{x=1}$.

Under some simple assumptions on the periodicity of the allocation $\lambda$ and the maintenance $f$ (see the Appendix B for details), the fraction of individuals spawning at time $t$ reaches a periodic steady state of unit period after the transients die out. This steadystate spawning pattern is designated $F^{\infty}(t)$. This spawning pattern represents the spawning behavior of the entire population and can be used to determine the coherence in spawning among individuals in the population. We numerically compute these spawning patterns for different biologically consistent allocation strategies and different maintenance cost functions using the computational approach detailed in the "Appendix B".

Entrainment, mode-locks, coherence width, quiescence, and spawn number

Spawning is defined to be entrained to the seasonal insolation cycle if, at steady state, there are a fixed number of spawning events in a given number of seasonal cycles (Gurney et al. 1992). Specifically, the coral is entrained to an $r: q$ mode-lock with positive integers 
$q$ and $r$ if there are exactly $q$ spawning events in a span of $r$ seasonal cycles, which in our model is equivalent to $q$ spawning events in $r$ years; intuitively, the coral is entrained to a harmonic of the insolation cycle. Then $q$ and $r$ characterize entrainment for a particular choice of coral model parameters. However, in general, corals only spawn once annually at a fixed time of year. Although there is some evidence of biennial spawning and asynchronous spawning (Mangubhai and Harrison 2008), there is no consensus on such phenomena among coral biologists, and hence, we do not consider these scenarios here. Therefore, we only consider mode-locks of the form $r: 1$. In other words, the coral spawns once every $r$ years and is entrained to the $r$ th harmonic of the driving insolation rhythm. If the corals were to lock on to an $r: q$ mode, there would be $q$ spawning events in $r$ years and the time within a year when spawning occurs would not be fixed.

Unfortunately, the above characterization of entrainment is only possible in the absence of noise when the time between successive firing times is deterministic. In the presence of fluctuations in the energy input, where a distribution of firing times is obtained, we instead define multiple characteristics of the steady-state firing pattern $F^{\infty}(t)$ in Eq. 9 to describe its entrainment properties. The coherence width $W$ (measured in days) measures how confined or focused spawning is to a certain time of the year (within one cycle of driving insolation rhythms):

$$
\begin{aligned}
W & =\sqrt{\frac{\int_{t}^{t+1}(\tau-\bar{T})^{2} F^{\infty}(\tau) d \tau}{\int_{t}^{t+1} F^{\infty}(\tau) d \tau}} \text { where } \\
\bar{T} & =\frac{\int_{t}^{t+1} \tau F^{\infty}(\tau) d \tau}{\int_{t}^{t+1} F^{\infty}(\tau) d \tau} .
\end{aligned}
$$

However, the coherence width does not specify whether no firing occurred during significant parts of the driving cycle. We want to capture the lack of spawning outside the spawning window observed in field experiments with corals. We term periods of negligible firing in the spawning pattern $F^{\infty}(t)$ as quiescence periods and look for allocation strategies that can produce significant quiescence periods in the spawning pattern.

When a coral achieves an $r: 1$ mode-lock, spawning occurs in 1 year and then skips the next $r-1$ years before occurring again. Such a skipping phenomenon is not captured by the previous measures. The fraction of the population spawning at time $t$ is composed of individuals that have already spawned varying number of times; we shall refer to the number of times an individual coral has spawned including a spawn in the current cycle as its spawn number $p$. Although we are not interested in the absolute spawn number of an individual, the spread of contributing spawn numbers of individuals in a certain cycle is a measure of if and how often coral individuals "skip" spawning cycles; a wide spread implies that corals often skip spawning cycles. We discuss the relevance of this skipping in the "Effect of parameter changes on spawning behavior".

\section{Entrainment in noiseless coral I\&F models}

The simplest bioenergetic model for a coral individual uses purely time-dependent allocation strategies and maintenance cost functions, i.e., $f\left(X_{t}, t\right)=f(t)$ and $\lambda\left(X_{t}, S_{t}\right)=\lambda\left(S_{t}\right)$. We show in "Appendix C" that perfect matching of the parameters of the model and the driving seasonal signal (its period and strength) is necessary for achieving the required entrainment. Therefore, entrainment in such scenarios lacks robustness, and the addition of the noise perturbs the system away from these optimal parameter values; we corroborate this fact with simulations in the "Simple reserve accumulator" section. As such, state-independent scenarios in the stochastic model are incompatible with observed spawning synchrony in corals.

Intuitively, a generic state-independent system does not involve any feedback or decisions based on the state of system in order to compensate for the effects of noise and the lack of robustness is understandable. State dependence of the internal dynamics or allocation strategy introduces some feedback within the system and has the potential of providing robust entrainment to the forcing signal.

In the presence of state-dependent maintenance, the I\&F models exhibit two types of behavior: entrainment of a particular mode $r: q$ or quasiperiodic oscillations (Coombes and Bressloff 1999; Keener et al. 1981). We now present a metric to determine which of these two behaviors is exhibited by the model for a particular parameterization. In the absence of noise, the time of the $n$th spawn $t_{n}$ completely determines the time of the $(n+1)$ th spawn $t_{n+1}$. The transition map $t_{n+1}=$ $T\left(t_{n}\right)$ that might be implicitly defined for some models provides a way of computing the sequence of spawning times. Starting from some initial time $t_{0}$, the $n$th firing time $t_{n}$ can be computed by recursively applying the map: $t_{n}=T^{(n)}\left(t_{0}\right)=T\left(T^{(n-1)}\left(t_{0}\right)\right), T^{(1)}\left(t_{0}\right)=T\left(t_{0}\right)$. For a map $T$ (.), we define its rotation number $\rho$ as the average phase gained for every cycle of the input (Chpt. 11, Katok and Hasselblatt 1996):

$$
\rho=\lim _{n \rightarrow \infty} \frac{t_{n}}{n}=\lim _{n \rightarrow \infty} \frac{T^{(n)}\left(t_{0}\right)}{n} .
$$




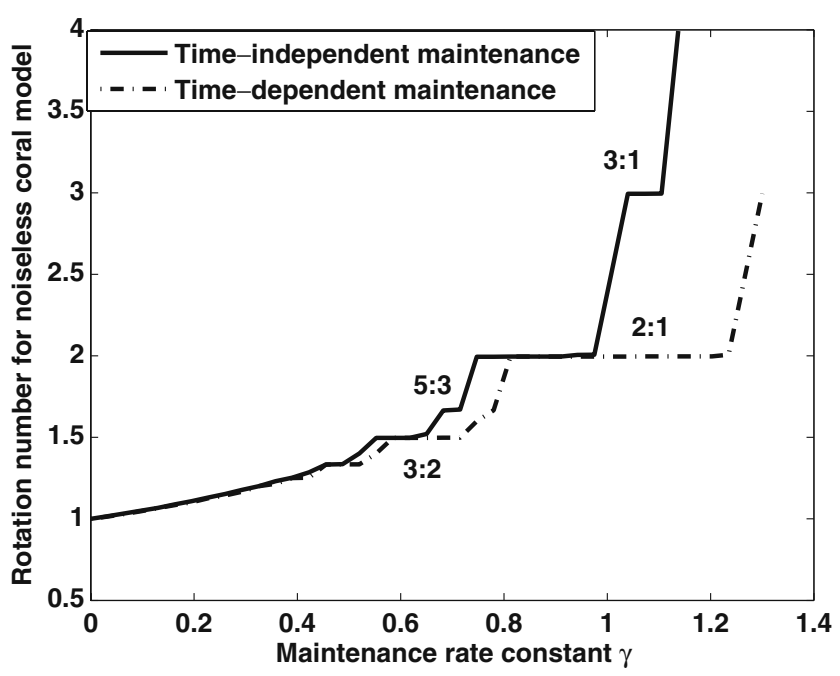

Fig. 2 The rotation number from Eq. 2 for the two models in the "Reserve accumulator with maintenance" section for $T_{0}=$ 0.75 . The flat sections of the curves correspond to regions of $r: q$ mode-locks and rational values of the rotation number $\rho=$ $q / r$. The intermediate values of $\rho$ (and $\gamma$ values) correspond to quasiperiodic oscillations. Outside this range of $\gamma$ values, the noiseless system does not reach threshold

Since the driving seasonal signal (insolation) has unit period, we can take the spawning time module 1 to be the firing phase within a year and the spawning phase angle can be obtained by multiplying by $2 \pi$. Henceforth, we interchangeably use spawning time and spawning phase.

It is known that this rotation number $\rho$ is independent of the initial conditions $t_{0}$. Moreover, the coral I\&F model achieves an $r: q$ mode-lock if $\rho=q / r$ and exhibits quasiperiodic firing if $\rho$ is irrational (Chpt. 11, Katok and Hasselblatt 1996). These quasiperiodic oscillations are space filling or in other words, given enough time, spawning occurs at every time within a year. For example, in Fig. 2, the rotation number is shown for a range of maintenance rate constants $\gamma$ for the time-independent $(m(t)=1)$ and time-dependent $\left(m(t)=1+\alpha-S_{t}\right)$ models presented in the "Reserve accumulator with maintenance" section with natural spawning period $T_{0}=0.75$. In addition to $r: 1$ modelocks, some undesirable $r: q$ mode-locks are also observed. This curve is different for other natural periods of the I\&F model and the characterization of the entrainment behavior of the noiseless I\&F model for different values of $\gamma$ and $T_{0}$ is possible by computing Arnold tongue (the Arnold tongues for the timeindependent maintenance model can be found in Fig. 3, Coombes and Bressloff (1999)).

The fundamental difference in the regions of locking with maintenance (state dependence) is that there exist intervals of parameter values that lead to the same mode-lock and are therefore robust. Thus, a population of heterogeneous individuals with different maintenance rates, but with locking parameters within the same interval, will similarly entrain to the seasonal driver. This robustness also makes entrainment in the presence of noise possible as perturbations to the system have a smaller effect on the dynamics. Moreover, in a heterogeneous population, individuals could have parameter values corresponding to different $r: 1$ locks but still lead to synchronous spawning with individuals skipping different sets of spawns.

\section{Allocations to reproduction consistent with coral biology}

In this section, multiple allocation strategies for an individual coral consistent with coral biology are discussed. Desirable allocation strategies are those that produce coral spawning patterns with coherence widths that are within the limits of the circa-lunar scale, i.e., $W \approx 30$ days. Based on our hierarchical timekeeping hypothesis, once coherence to within the circa-lunar scale is achieved, processes acting on the circa-lunar and subsequently circa-diel scales can determine the precise time of spawning observed in the field.

We probe each biologically consistent allocation strategy via numerical solution to the model in Eq. 7; to the best of our knowledge, this model has no closedform solution for time-dependent drift or diffusion factors. The list of parameters in our model and choice of parameter values are detailed in Table 1; these default parameter values are used unless otherwise mentioned.

Simple reserve accumulator

The first class of models introduced in this section represents corals with no maintenance, i.e., $f\left(X_{t}, t\right) \equiv 0$.
Table 1 Parameters in the I\&F model with the nominal parameter values

\begin{tabular}{lll}
\hline Parameter & Description & Value \\
\hline$T_{0}$ & Natural period of I\&F model and length of reproductive cycle in years & 0.75 \\
$\gamma$ & Maintenance rate constant & 0.5 \\
$\mathrm{CV}$ & Coefficient of variation of fluctuations in insolation as a fraction of $S_{0}$ & 0.1 \\
$\alpha$ & Magnitude of seasonal variation in mean insolation & 0.5 \\
$\phi$ & Sets location of peak insolation at $\phi+\frac{\pi}{2}$ & $0.5 \pi$ \\
\hline
\end{tabular}




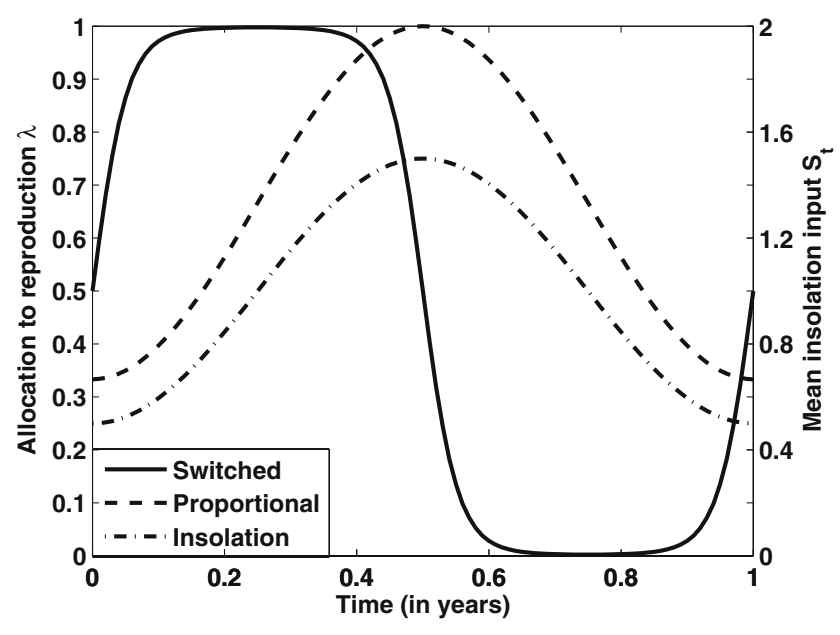

Fig. 3 Two possible allocations to reproduction $\lambda\left(X_{t}, S_{t}\right)$ along with the mean insolation energy input rate $S_{t}$, where the organism has knowledge of time only through the seasonal insolation variation

The bioenergetic implications of this model structure are that the energy allocated to reproduction entirely leads to augmentation of the reserves without any maintenance costs, i.e., incoming energy $\Delta x$ causes the reserves to increase by the same $\Delta x$. We wish to verify the results of our analysis from the "Entrainment in noiseless coral I\&F models" section that tight coherence in spawning is not achievable in these stateindependent models and allocations.

\section{Switched allocation}

The coral commits to reproduction only during periods of increasing energy input. This strategy ensures that the coral has some certainty that there is increased energy available at least in the near future. We formulate this allocation as $\lambda\left(X_{t}, S_{t}\right)=0.5+0.5 \tanh \left(c S_{t}^{\prime}\right)$ ) (see Fig. 3), where the constant $c$ controls the switching transition time between "off" $(\lambda=0)$ and "on" $(\lambda=$ 1). The spawning pattern produced by this switched allocation is shown in Fig. 4a. Although this allocation produces spawning quiescence during a significant part of the year, the coherence width of spawning spans several months and is not desirable. Figure $4 b$, c is two possible representations of the distribution of spawning times for four successive firings of coral individuals as a probability density and a cumulative distribution, respectively. We designate an arbitrary spawn event as the reference and consider, among those individuals that participated in the reference spawn, the distribution of times when those individuals spawned for the first subsequent $(\mathrm{p}=1)$ and second subsequent $(\mathrm{p}=2)$ times, and so on. We observe that, within each spawn number, spawning is spread across about five insolation cycles.

The quiescence in the spawning pattern in Fig. 4a corresponds to the flat sections of the cumulative distribution of each spawn number in Fig. 4c. The cumulative distributions can be interpreted as the fraction of the individuals that spawn for the $p$ th time before time $t$ on the $x$-axis. Very narrow periods of relatively high spawning are revealed as sharply increasing cumulative distribution curves. Due to the clarity of the cumulative distributions over the probability distributions in Fig. $4 \mathrm{~b}$, henceforth we shall only show plots similar to Fig. $4 \mathrm{c}$ for the various models.

We strive to obtain allocations whose cumulative distributions have two highly desirable attributes (a) wide flat regions representing quiescence and (b) rapidly a

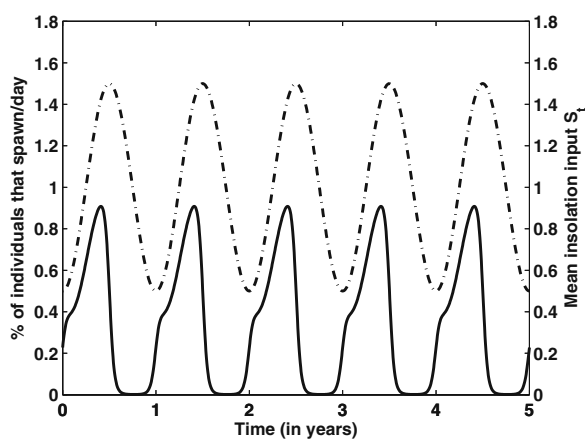

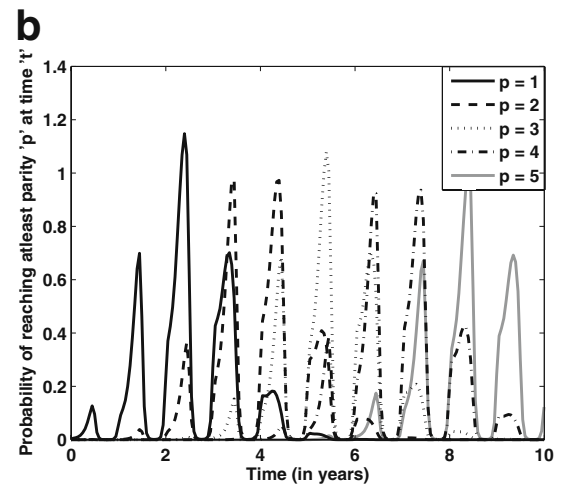

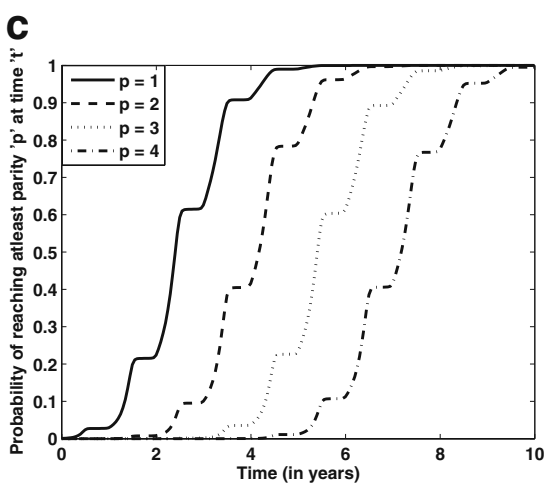

Fig. 4 a The percentage of the individuals in a colony that spawn in a day shown for a 5-year period for the simple reserve accumulator under $\lambda\left(X_{t}, S_{t}\right)=0.5+0.5 \tanh \left(3 S_{t}^{\prime}\right)$. The normalized environmental driver $S_{t}$ is shown as a dash-dot curve. b Contribution of individuals of different spawn number or number of reproductive events to the total output per day shown for successive spawn numbers. c The cumulative probability distribution of achieving at least a spawn number $p$ within time $t$ shown on the $x$-axis 
increasing curves connecting those flat regions representing the narrow spawning periods (in the extreme we want a step function). Note that the spawning pattern and the cumulative distribution are two different ways of visualizing the same data.

It appears that although the switched allocation introduces quiescence into the firing pattern, spawning at other times is not sufficiently coherent. The coherence width is 65.66 days, which encompasses two lunar cycles and, hence, insufficient to unambiguously determine the day and time of spawning using the circa-lunar and circa-diel scales.

\section{Proportional allocation}

Another variant of the previously described switching allocation is the following model that we term the proportional allocation (see Fig. 3): $\lambda\left(X_{t}, S_{t}\right)=S_{t}$. The corals adopting this strategy allocate opportunistically to reproduction, i.e., they assign increased amounts of energy to reproduction if they have extra energy at hand and vice versa.

The spawning pattern for the proportional allocation in Fig. 5a does not show any quiescence; there is always a basal number of individuals spawning and is confirmed by the cumulative distribution in Fig. 5b. The coherence width is approximately 72 days, which is also significantly larger than our requirements. Notice the lack of sharp coherence in spawning times is corroborated by the gently rising curves in Fig. 5b. In Fig. 5c, the progression of reserve level for one individual over time is shown. The reserves build up to 1, fire, and are then reset to 0 . The feature of coral individuals skipping insolation cycles is clearly seen in the progression from the second to the third spawning in Fig. 5c. In summary, this allocation is in many ways even less desirable than the switched allocation.

\section{Reserve accumulator with maintenance}

We consider in this section systems with state- and possibly time-dependent maintenance costs to augmenting the reproductive reserves $X_{t}$.

\section{Proportional allocation with time-independent linear maintenance}

In order to understand the effect of state-dependent maintenance, we consider a model with the simple proportional allocation strategy. The maintenance on the reserves is proportional to the current reserve level: $f(x, t)=\gamma x$. Thus, it costs progressively more energy to raise the levels by the same amount as reserves near their full capacity. Such a maintenance would represent the biological situation where successive advanced stages of gamete production and maturation required more energy for the same relative progress through a stage.

The spawning pattern in Fig. 6a has a coherence width of about 49 days, which is a significant improvement over the state-independent models discussed previously. The quiescence seen as flat regions and reasonably rapid increases in the cumulative distribution curves in Fig. $6 \mathrm{~b}$ confirms that this model is more biologically plausible. However, the number of seasonal cycles a coral skips under this model is about 4 (can be seen from the number of seasonal cycles that each of the cumulative distribution curves in Fig. $6 \mathrm{~b}$ a

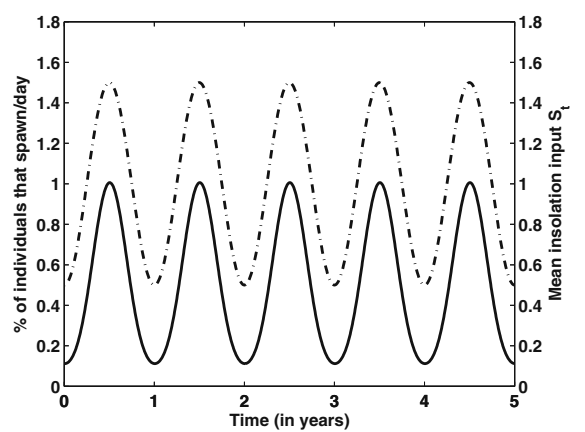

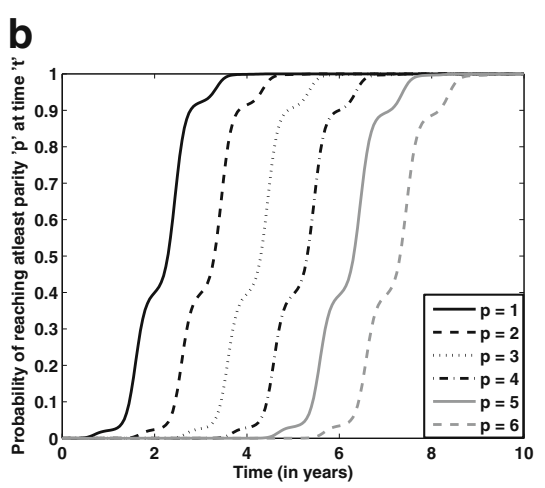

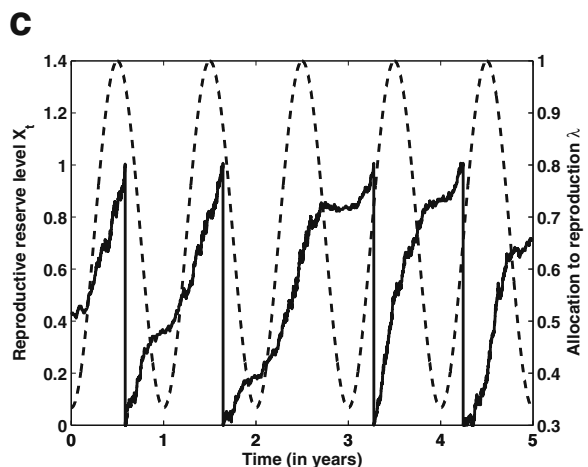

Fig. 5 a The percentage of the individuals in a colony that spawn in a day shown for a 5-year period for the simple reserve accumulator under $\lambda\left(X_{t}, S_{t}\right)=S_{t}$. The normalized environmental driver $S_{t}$ is shown as a dash-dotted curve. b The cumulative probability distribution of achieving at least a spawn number $p$ within time $t$ shown on the $x$-axis. c The progression of state $X_{t}$ is shown for one coral individual under the proportional allocation along with the allocation $\lambda$ (dashed curve) as a function of time 
a

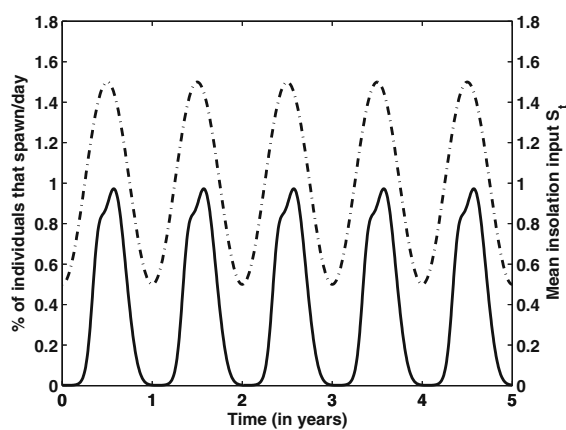

b

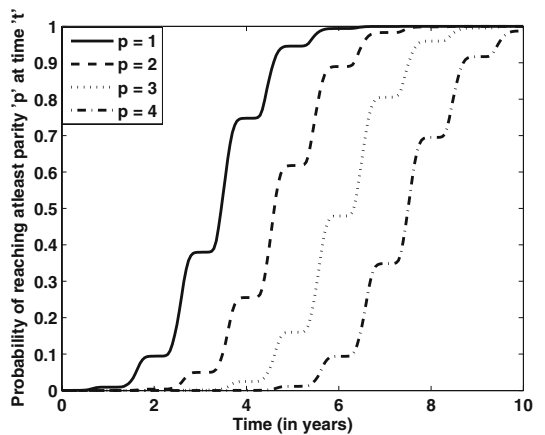

C

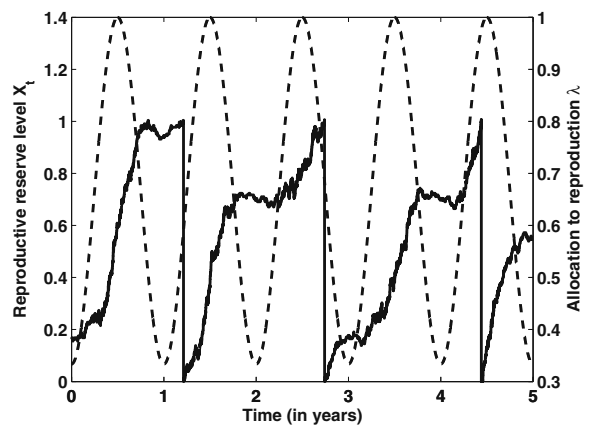

Fig. 6 a The percentage of the individuals in a colony that spawn in a day shown for a 5-year period for the reserve accumulator with maintenance under $\lambda\left(X_{t}, S_{t}\right)=S_{t}$ and $f(x, t)=\gamma x$. The normalized environmental driver $S_{t}$ is shown as a dash-dotted curve. b The cumulative probability distribution of achieving at

spans). This spread is larger than the spread for the corresponding model with no maintenance.

\section{Proportional allocation with time-dependent (seasonal) linear maintenance}

This model retains the proportional allocation strategy as the previous model, but the maintenance costs are seasonally varying (time dependent). It has been hypothesized that SST affects the rate of gametogenetic development of corals. Assuming a perfect correlation between higher SST temperature and insolation (in reality, peak SST lags peak insolation), we assume the maintenance cost to commit to reproduction is higher at lower temperatures. Therefore, maintenance costs least a spawn number $p$ within time $t$ shown on the $x$-axis. $\mathbf{c}$ The progression of state $X_{t}$ is shown for one coral individual under proportional allocation along with the allocation $\lambda$ (dashed curve) as a function of time

are negatively correlated with SST resulting in $f(x, t)=$ $\gamma\left(1+\alpha-S_{t}\right) x$.

This model produces sharper spawning patterns than those introduced earlier, as seen in Fig. 7a, and is corroborated by a step-like cumulative firing distribution in Fig. 7b. The mechanism behind the success of this model is seen from the time trace of the state in Fig. 7c. The resource level does not grow during periods of low and decreasing energy input and the capability to exceed the threshold is restricted to periods of high insolation. The spawning pattern in Fig. 7 also shows some signs of permitting a split spawn (observe the distinct double peak), in which spawning output is split between two successive full moons during the spawning period. This, however, appears to occur only during years where the full moon occurs very early in the a

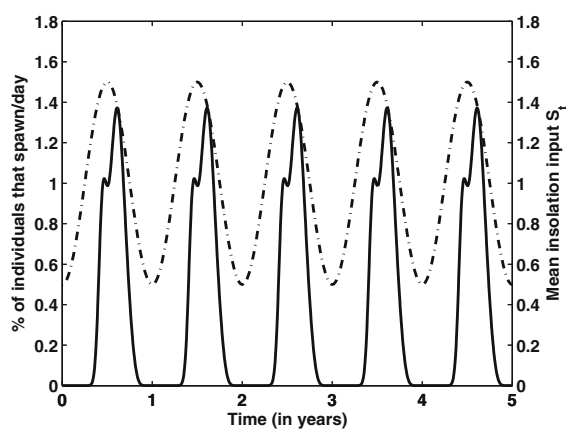

b

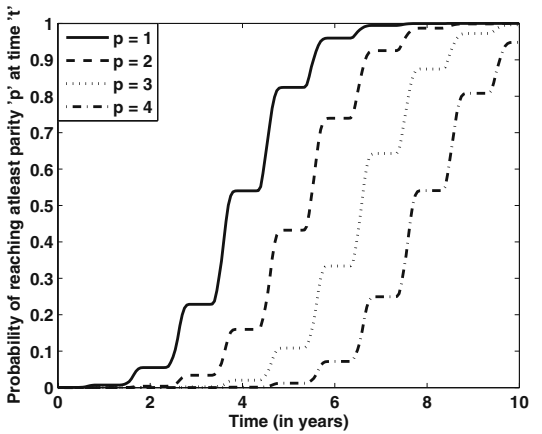

C

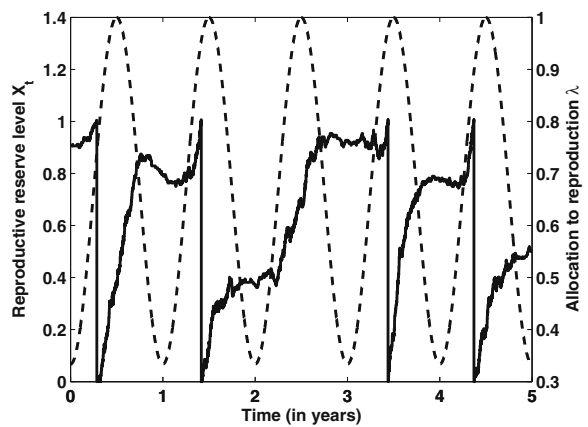

Fig. 7 a The percentage of the individuals in a colony that spawn in a day shown for a 5-year period for the reserve accumulator with maintenance under $\lambda\left(X_{t}, S_{t}\right)=S_{t}$ and $f(x, t)=\gamma(1+\alpha-$ $\left.S_{t}\right)$. The normalized environmental driver $S_{t}$ is shown as a dashdotted curve. $\mathbf{b}$ The cumulative probability distribution of achiev- ing at least a spawn number $p$ within time $t$ shown on the $x$-axis. c The progression of state $X_{t}$ is shown for one coral individual under proportional allocation along with the allocation $\lambda$ (dashed curve) as a function of time 
month long "spawning window" allowing for another full moon at the end of the period (Bastidas et al. 2005).

\section{Effect of parameter changes on spawning behavior}

In the previous section, we presented the output of the bioenergetic models obtained from numerical computation of the model. Such analyses nevertheless do not provide a complete understanding of the dynamics of the model particularly with regard to other model parameterizations. We present here the performance of the stochastic models from the "Reserve accumulator with maintenance" section for different maintenance rates and strength of the environmental fluctuations. We wish to investigate if the noiseless system dynamics provides insight into the stochastic performance.

Our hypothesis is that the circa-annual rhythms act as the ultimate trigger and prepare the corals for spawning within one lunar cycle in order for the circa-lunar and circa-diel drivers to determine the 'correct' night of spawning. In Fig. 8, the coherence width is shown for different maintenance rates for the proportional allocation with time-independent and time-dependent linear maintenance costs. The most striking feature of the stochastic model performance is the smooth variation of coherence width over all values of $\gamma$ distinct from the noiseless dynamics in Fig. 2, where specific intervals were obtained for different mode-locks. The coherence in spawning improves for increasing maintenance rates monotonically across different noise intensities.

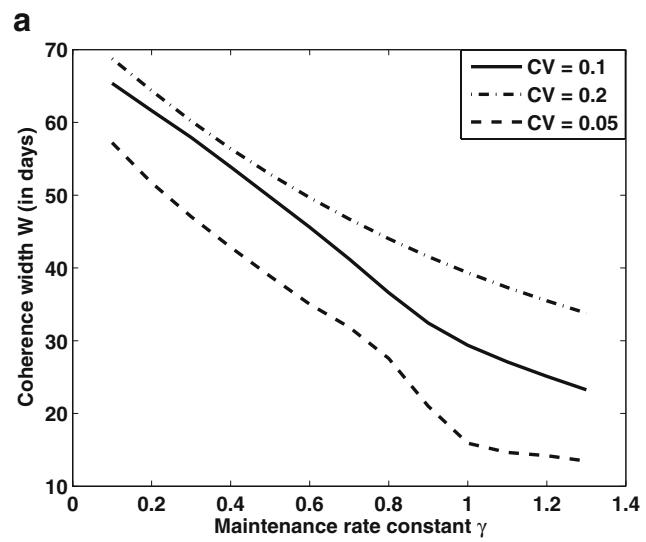

Fig. 8 a (Time-independent maintenance) The coherence width of the entrained spawning pattern for proportional allocation with linear time-independent maintenance as a function of the maintenance rate constant $\gamma$ for three different intensities of fluctuation in the insolation. b (Time-dependent maintenance)
We also observe in Fig. 8a, b that the performance is insensitive to the fluctuation intensities for small maintenance costs, but performance decreases with increasing noise at $\gamma$ values close to 1 . We believe that this is the direct result of the noiseless dynamics in Fig. 2, where the region of different $r: 1$ mode-locks for both models is seen to lie between 0.8 and 1.2. In these mode-locking regions, noise introduces perturbations about the fixed point of the noiseless map, and hence, there is a "linear" relationship between the coherence width and noise intensity. At smaller $\gamma$ values, mostly quasiperiodic oscillations are seen in the noiseless system (from the irrational rotation numbers), and the spread in the spawning pattern is a direct result of quasiperiodicity, which exists even in the absence of noise. In addition, the stochastic nature of the system causes different individuals to experience slightly different parameter values (for e.g., maintenance rate values $\gamma$ ), and this results in the lack of sharp boundaries between different dynamics as seen in the noiseless system.

The only difference in performance between the time-independent and time-dependent models is observed at low maintenance rates, where the model with time-dependent maintenance (Fig. 8b) has a smaller coherence width across the entire range of noise intensities. On the other hand, the model with timeindependent maintenance (Fig. 8b), while not significantly worse, has more noise intensity sensitive performance.

In Fig. 9, we test our earlier conjecture on a system with time-independent maintenance and $T_{0}=0.65$ that b

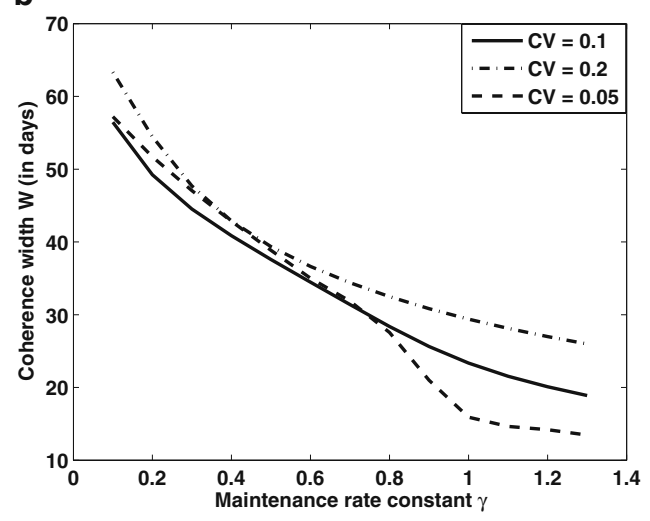

The coherence width of the entrained spawning pattern for proportional allocation with linear time-dependent maintenance as a function of the maintenance rate constant $\gamma$ for three different intensities of fluctuation in the insolation 


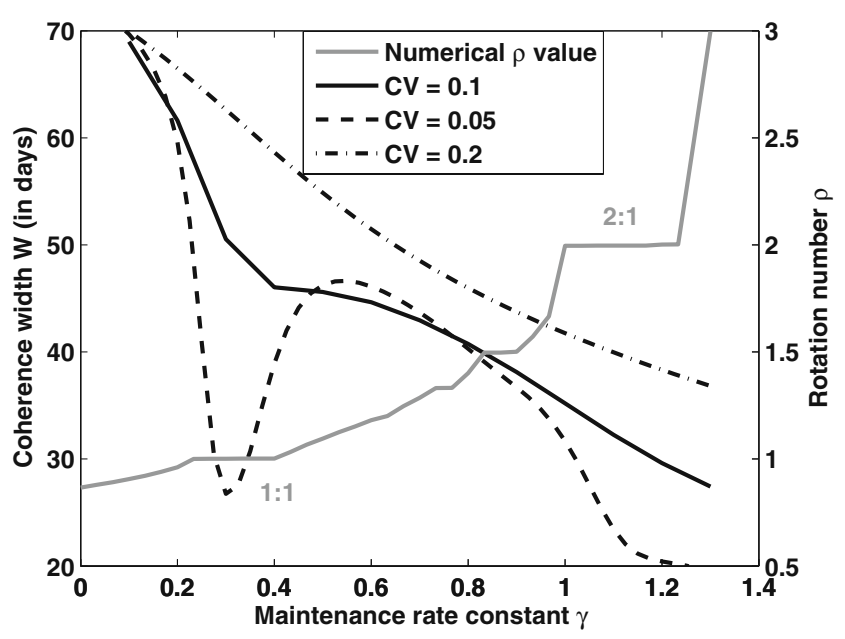

Fig. 9 The coherence width obtained for the stochastic timeindependent linear maintenance model for different maintenance rates for a different natural period of the noiseless I\&F model $\left(T_{0}=0.65\right)$. The rotation number of the corresponding noiseless system is shown as a gray solid line. The regions of maintenance rates when $r: 1$ mode-locking is shown by the noiseless system are regions where the stochastic system performance is most sensitive to variation in noise intensity

regions of parameter space, where the noiseless system shows $r: 1$ mode-locking, are most sensitive to the choice of noise intensity as perturbations in the input cause "linear" changes in the output. The figure shows that the regions of parameter space that show $1: 1$ and $2: 1$ mode-locking are sensitive to the noise intensity whereas the coherence width for the spawning pattern is similar in regions with quasiperiodic oscillations (irrational rotation number).

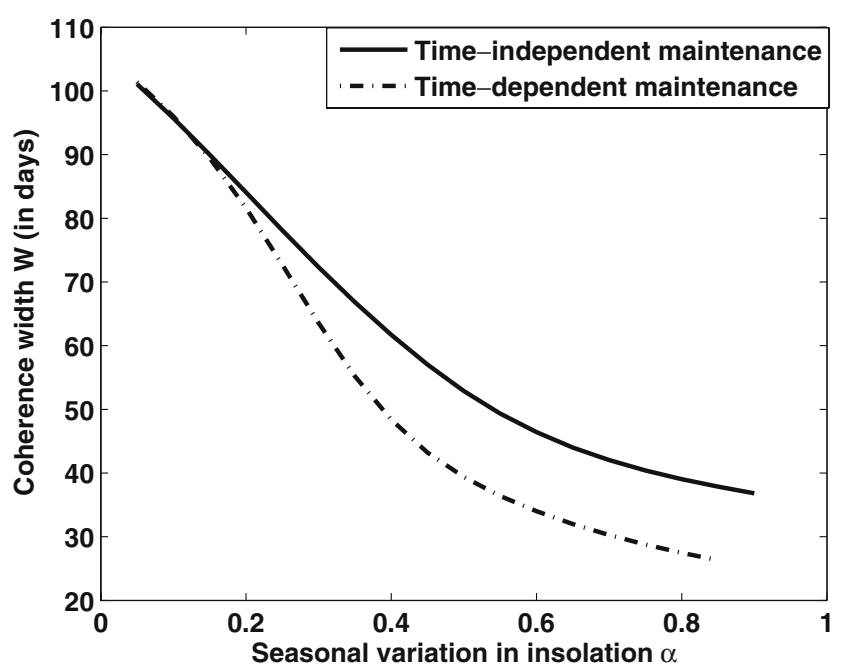

Fig. 10 The coherence width obtained for the stochastic time-independent and time-dependent maintenance models for different magnitudes of the seasonal insolation variation $\alpha$ for maintenance rate constant $\gamma=0.5$
Finally in Fig. 10, we consider the effect of strength of the seasonal insolation variation on the coherence width in spawning. As expected, we observe that a stronger seasonal driver leads to higher spawning coherence in both the time-dependent and timeindependent maintenance models. The only difference between the two models arises from the marginally better overall coherence achieved by the time-dependent maintenance model (see Fig. 8).

\section{Discussion}

We showed that simple allocation strategies can result in spawning once a year with coherence width within the resolution of the finer timing mechanisms in the circa-lunar and circa-diel scales and that energy considerations possibly play a direct role in the entrainment of coral spawning cycles. Allocation strategies that were independent of the current reproductive state of the coral require perfect matching of the model (and its parameters) to the forcing rhythm (insolation), i.e., the sets of parameters that lead to entrainment is a set of measure zero even in the noiseless scenario. On the addition of noise, the system is perturbed away from this optimal matching resulting in poor coherence and lack of quiescence in spawning. These factors make such purely time-dependent strategies extremely non-robust and, hence, unlikely as a class of mechanisms operating in biological situations, where populations tend to be heterogeneous due to genetic and environmental factors.

It is understandable that strategies that do not correct for the perturbations caused by noise based on the current state of the system, i.e., systems without feedback, would not be robust. We therefore explored state-dependent models that had a maintenance cost that depended linearly on the state of the system. This would be biologically associated with systems where the same percentage of progress through a stage costs progressively more in later stages. Entrainment of different modes could be observed over significant ranges of parameter space, even in the absence of noise. In the stochastic system, coherent spawning with coherence width of the order of a month and quiescence were observed. With significant regions of parameter space permitting the same $r: 1$ mode-lock, these models accommodate heterogeneity in a population of individuals as is common in nature. This suggests that a strong regulatory mechanism exists for the management and commitment of energy toward reproduction, an observation made by Giese $(1959,1966)$ on a class of marine invertebrates. 
While the noiseless system shows mode-locking of the expected mode over distinct subsets of parameter space, the stochastic system shows coherent spawning over a wide range of maintenance rate values in which the noiseless system with constant input achieved the spawning threshold. Moreover, the coherence width of spawning progressively decreased with increased maintenance rate. This seems to suggest that more synchronized spawning can be achieved by higher mode-locks where individuals skip cycles and spawn once every $r$ cycles of the driver. In other words, corals can tighten their spawning coherence and improve their likelihood of reproductive success at the cost of expending more energy towards reproduction. Thus, there is a tradeoff between synchrony (and spawning success) and the total energy investment per spawn.

The regions that showed $r: 1$ mode-locks in the absence of noise, however, responded differently to changes in the noise intensity. Regions of noiseless entrainment showed linear dependence of the spawning coherence on noise intensity. These regions correspond to a fixed point on the spawning map about which the system can be linearized and thus explain this dependence of coherence width on the noise intensity. In this sense, these regions of noiseless entrainment are robust as they provide "good" performance when the driving energy conditions are "good" and vice versa. In regions of quasiperiodicity, the noiseless system displays wide variation in the spawning times from cycle to cycle and, therefore, has a spread of spawning times even in the absence of noise. Thus, no significant effect of noise is seen over the already present spread of spawning times.

Stochasticity also helps to genetically mix a population entrained to an $r: 1$ mode-lock; in its absence, individuals that are together in an $r: 1$ mode-lock always spawn together and never spawn concurrently with others that are in $r: 1$ locks but in a different skipping pattern. Such a mechanism that favors greater genetic mixing might also be favored by evolution. Several hypotheses support synchronized spawning as the evolutionarily favored mechanism including higher likelihood of successful cross-fertilization, predator satiation, and genetic diversity. In the related work on masting, Yamauchi (1996) analyzed the evolutionary basis for synchronized seeding. Although similar analyses could be applied to study both the coherence and intermittency in spawning, such a study is beyond the scope and focus of this work.

We only considered maintenance rate values that resulted in spawning in the noiseless scenario. We know that a stochastic I\&F system that does not reach the threshold exhibits stochastic resonance and the coher- ence width of spawning is minimum at an optimal noise intensity (Plesser and Tanaka 1997). Thus, tight spawning coherence in an I\&F system can still be achieved outside the range of parameter values we consider. In this scenario, the organism accumulates energy to a subthreshold level and waits for a burst of fluctuations to move it across threshold. This is a possibility that we have not addressed and could have possible relevance in synchronizing spawns, but we are unaware of any experimental evidence pointing towards such a mechanism.

We assumed that the insolation rhythms were mean shifted sinusoids. This does not accurately represent the mean insolation at many geographical locations. In fact, this representation is quite inaccurate within the tropics where the peak insolation profile is bimodal corresponding to the vernal and autumnal equinoxes. The peak insolation (in the absence of other atmospheric effects) for a reef at $15^{\circ} \mathrm{N}$ latitude input into the time-independent linear maintenance model results in a coherence width of about 45 days as seen in Fig. 11. Although performance is worse than with a sinusoidal input, we can potentially improve performance with an appropriate change in the model.

We stated in the introduction that SST is inconsistent with observed coral spawning data (Baird et al. 2009). Nevertheless, SST can successfully explain the 1-month difference between inshore and offshore spawning in the Great Barrier Reef (Willis et al. 1985). Therefore, in Fig. 12, we test SST-induced coherence in spawning

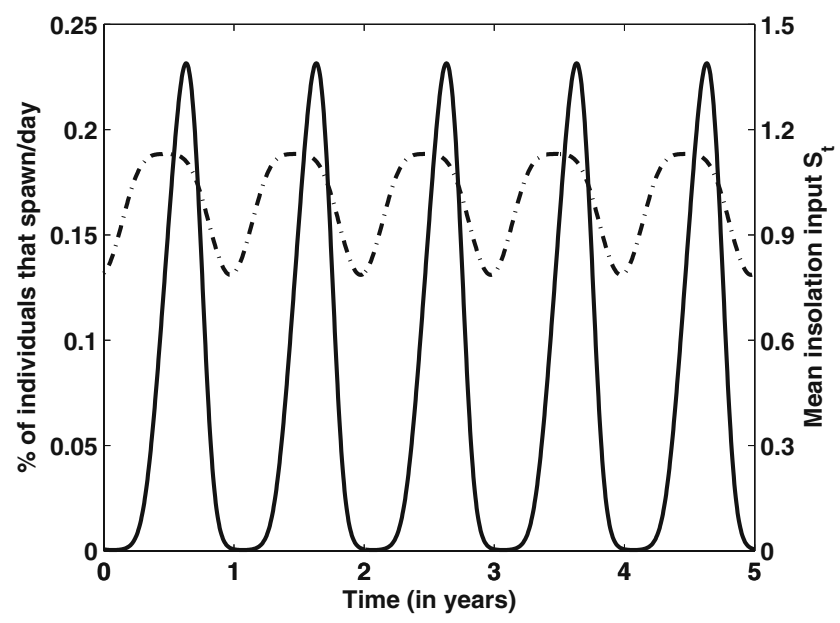

Fig. 11 The spawning pattern from the time-independent linear maintenance model $m(t)=1$ for the maximum insolation profile at $15^{\circ} \mathrm{N}$, which is shown as the dash-dotted curve, for maintenance rate constant $\gamma=1.5$ (we use a higher maintenance rate constant to achieve a coherence width of about one lunar cycle). The coherence width is 45 days 


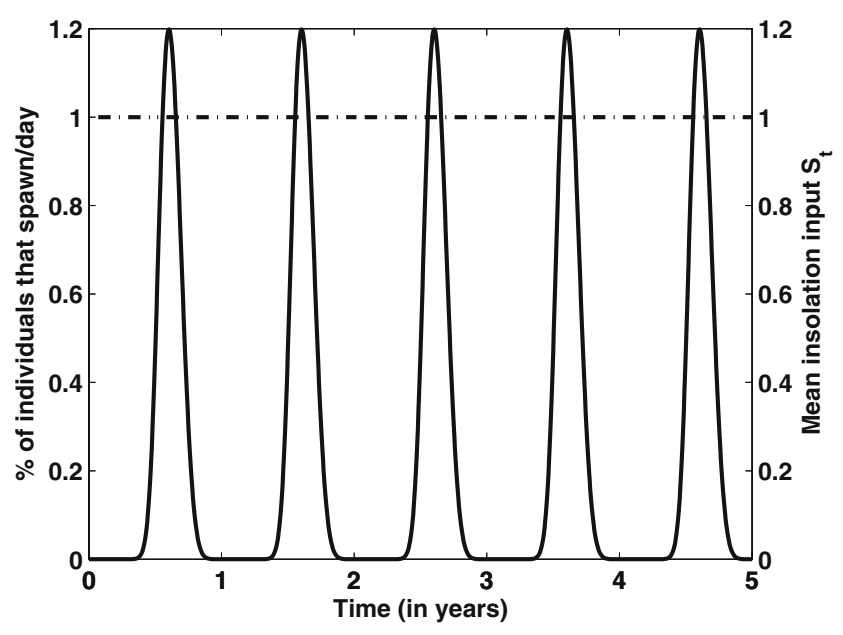

Fig. 12 The spawning pattern for a 5-year period for a coral individual under constant light (energy input) conditions with maintenance cost varying in accordance with SST as in the time-dependent linear maintenance model, i.e., $\lambda\left(X_{t}, S_{t}\right)=1$ and $f(x, t)=\gamma(1-\tilde{S}(t)) x$. The environmental driver $S_{t}$ is shown as a dash-dotted curve

under constant insolation (energy input) conditions. Under constant insolation, coral individuals incur higher maintenance costs at lower temperatures corresponding to slower metabolic processes, as in the timedependent maintenance model. We can see that our model produces weakly coherent (coherence width of 84 days) spawning. Nevertheless, we cannot completely rule out the influence of SST on spawning synchrony. For instance, the time-dependent maintenance model that produces tight synchrony is an instance of synergy between two circa-annual drivers: insolation and SST.

We are currently performing quantitative analyses to measure the level of an egg-specific protein vitellogenin in Acropora digitifera samples from the Republic of Palau as a function of time before spawning for use as a marker for the various stages of reproductive development, much like the reserve state that we model. These data can be fitted to the individual traces of state versus time shown in the "Allocations to reproduction consistent with coral biology" section and used for model discrimination and hypothesizing modified strategies. Another possibility would be to use diameter (size) of gametes (or gonadal index) (Giese 1959) as a measure of the stage of reproductive development as is often used by researchers studying gametogenetic cycles in corals and other spawning marine invertebrates. The limited data available on gamete diameter versus time (Fig. 5, Vargas-Ángel et al. 2006) appear to correlate well with trends seen in the traces of state shown here. Moreover, we have assumed here that coral individuals retain their residual gametes during the quiescence periods and continue development when favorable energetic conditions are reached. While coral fragments are known to reabsorb unreleased gametes after the spawning season, there is insufficient experimental evidence to support or contradict our assumption, although this merits further investigation.

We have posited here that insolation is the primary energetic driver in photosymbiotic corals. Insolation is known to be the primary driver of primary productivity in marine and aquatic ecosystems. Hence, reproduction in many broadcast spawning marine invertebrates typically follows this driver (Starr et al. 1990); clams that filter-feed on micro-algae typically begin gametogenesis in response to the blooms of these algae (Braley 1984), just as abalones and urchins that graze on kelp begin gametogenesis after these macro-algae resume their growth cycles. Other examples of synchronously spawning marine coral reef invertebrates can be found in Babcock et al. (1992). Variants of our model may thus be applicable to a larger class of spawning invertebrates than corals.

However, there are species-, system-, and locationspecific phase delays in primary productivity that depend on local cycles of temperature, upwellings, and delivery of macro- and micronutrients. In the case of corals, it can be argued that the coral animal's homeostasis buffers the endosymbionts from exogenenous ocean variability in nutrients that normally limit algal productivity and damps the influence of these secondary environmental factors that govern local primary productivity. This homeostasic buffering is not perfect and our models can explain the observed deviation of spawning dates of specific populations of corals (and other marine invertebrates) from strict calendar synchrony with other populations of the same species in the same hemisphere-a previously unresolved puzzle.

Acknowledgements We would like to particularly thank Charlie Boch for enriching our understanding of coral biology especially through his extensive experimental work in the field. We also acknowledge numerous useful discussions with Erik Muller, Laure Pecquerie, Alison Sweeney, Pete Edmunds, and Aileen Morse. This work is supported by the Institute for Collaborative Biotechnologies through Grant W911NF-09-D-0001 from the US Army Research Office and National Science Foundation under Grant EF-0742521.

Open Access This article is distributed under the terms of the Creative Commons Attribution Noncommercial License which permits any noncommercial use, distribution, and reproduction in any medium, provided the original author(s) and source are credited. 


\section{Appendix A: Stochastic differential equation model of a coral individual}

The average rate at which photosynthate is produced by the Symbiodinium from the incident insolation is $S_{t}$. The short-term fluctuations in energy input are modeled as additive Gaussian noise in the energy input. The state of each coral is a nonnegative, stochastic variable $X_{t}$ representing the total energy invested in the reproductive apparatus. The reproductive reserve $X_{t}$ is normalized such that the coral spawns when $X_{t}=1$ and the reserves are reset to zero after spawning.

A fraction, $\lambda\left(X_{t}, S_{t}\right)$, of the energy received from the Symbiodinium is allocated to the reproductive reserves (see Fig. 1). The energy cost to sustain a certain quantity of reproductive reserves is $f\left(X_{t}, t\right)=\gamma f(t) X_{t}$. Note that maintenance costs are present even when there is no input, and this could result in the loss of reserves to maintenance under this formulation, but maintenance always goes to zero as the reserves go to zero. The complete dynamics of the reserves for a coral individual is given by the following stochastic differential equation (in the Itô formulation):

$$
d X_{t}+\overbrace{\gamma f(t) X_{t} d t}^{\text {maintenance costs }}=\lambda\left(X_{t}, S_{t}\right) \overbrace{\left[S_{t} d t+\sqrt{2 D_{0}} d B_{t}\right]}^{\text {periodic environmental input }}
$$

with the positivity constraint $X_{t}>0$ and with the reset condition $X_{t}=\left.1 \Rightarrow X_{t}\right|_{t=t^{+}}=0$.

Rearranging the terms, we get

$$
\begin{aligned}
d X_{t} & =\left(-\gamma f(t) X_{t}+\lambda\left(X_{t}, S_{t}\right) S_{t}\right) d t+\sqrt{2 D_{0} \lambda^{2}\left(X_{t}\right)} d B_{t} \\
& \equiv h\left(X_{t}, t\right) d t+\sqrt{2 D\left(X_{t}, t\right)} d B_{t}
\end{aligned}
$$

where $2 D_{0}$ is the intensity of the Brownian motion ${ }^{2}$ and the time dependence of variables is denoted by subscripts for clarity. Since all the incoming energy is typically not allocated to reproduction due to other requirements such as growth and somatic maintenance, the fractional allocation is constrained as follows:

$0<\lambda<\lambda_{\max }<1$.

In the absence of maintenance costs $f\left(X_{t}, t\right) \equiv 0$ and constant energy input $S_{t}=$ constant, the mean frequency of firing of the noiseless $I \& \mathrm{~F}$ model is assumed to be $T_{0}$ (assuming the maximum fraction $\lambda_{\max }$ is allocated). Since the energy in reproductive reserves

\footnotetext{
${ }^{2}$ Brownian motion $B_{t}$ is a stochastic process whose value is the sum of discrete jumps in value, each of which is uncorrelated with all the prior jumps (van Kampen 2007). The value of each jump is normally distributed with variance directly proportional to the time interval between jumps.
}

represent the state of the gametogenetic cycle, which has a period of 3-9 months as outlined earlier, we expect $T_{0}<1$. The constant energy input $S_{0}$ that fills the reserves of unit capacity in time $T_{0}$ can be computed using

$1=\int_{0}^{T_{0}} d X_{t}=\int_{0}^{T_{0}} \lambda_{\max } S_{0} d \tau \Rightarrow S_{0}=\frac{1}{\lambda_{\max } T_{0}}$.

The solar insolation is a sinusoid with a mean of $S_{0}$ with a period of 1 year (idealized insolation in energy equivalents):

$S_{t}=S_{0}\{1+\alpha \sin (2 \pi t-\phi)\}, 0<\alpha<1 ; \quad \int_{0}^{1} S(\tau) d \tau=S_{0}$.

Without loss of generality, we can reduce the number of parameters in our model by defining the normalized allocation, normalized insolation input, and coefficient of variation of the fluctuations about the mean levels, respectively by:

$\tilde{\lambda}\left(x, S_{t}\right)=\frac{\lambda\left(x, S_{t}\right)}{\lambda_{\max }}, \tilde{S}_{t}=\frac{S_{t}}{S_{0}}$ and $\mathrm{CV}=\frac{\sqrt{D_{0}}}{S_{0}}$.

We can now rewrite Eqs. 3 and 4 as

$$
\begin{aligned}
d X_{t}= & \underbrace{-\gamma f(t) X_{t} d t+\frac{1}{T_{0}} \tilde{\lambda}\left(X_{t}, S_{t}\right) \tilde{S}_{t} d t}_{h\left(X_{t}, t\right) d t} \\
& +\underbrace{\sqrt{2 \mathrm{CV}^{2} \frac{\tilde{\lambda}^{2}\left(X_{t}, S_{t}\right)}{T_{0}^{2}}}}_{\sqrt{2 D\left(X_{t}, t\right)}} d B_{t} .
\end{aligned}
$$

We drop the $\tilde{\text {., }}$ and use $\lambda$ and $S_{t}$ to represent the normalized allocation and normalized insolation input respectively throughout this manuscript.

\section{Appendix A.1: Related work on I\&F models}

Equation 4, with the reset condition when reserves reach a value one, represents a generalization of the sinusoidally forced noisy I\&F model that has been studied extensively as a neuron model (Bulsara et al. 1996; Engelbrecht and Mirollo 2009). The dynamics exhibited by the I\&F model can be divided into two classes depending on whether the unforced system leads to "firing" (a threshold crossing of the state) or not. When the unforced system (in the absence of input) does reach the threshold, the conditions for $r: q$ entrainment and regions of quasiperiodic firing have been completely characterized for the leaky I\&F model in Coombes and Bressloff (1999) and Keener et al. (1981). 
When the system does not reach the threshold and, therefore, does not "fire", the system can exhibit stochastic resonance. Under resonance, the noise in the system can lead to threshold crossing and the smallest coherence width in the firing occurs at an optimal value of the noise intensity. The stochastic resonance phenomena as applicable to noisy sinusoidally forced I\&F models is discussed in Bulsara et al. (1996). The fundamental mathematical difference between the I\&F models in the literature and the model we propose is the positivity constraint that does not allow the state variable to take negative values. This additional constraint also makes the Fokker-Planck equation (7) for this model difficult to solve analytically.

\section{Appendix B: Computing spawning patterns using the Fokker-Planck formulation}

The spawning behavior of a population of coral individuals with reserve dynamics in Eq. 6 can be represented using the Fokker-Planck equation on the fraction of individuals $P(x, t)$ with reserves $X_{t}=x$ at time $t$. The equivalence of the Fokker-Planck and the Itô equation in Eq. 6 (Risken 1996) is often invoked to obtain the time progression of the probability distribution of state $X_{t}$, which is $P(x, t)$. Thus, under the assumption of noninteracting corals, the Fokker-Planck equation equivalent of the stochastic bioenergetic model is exactly the distribution of the state of reserves in the population:

$\frac{\partial P(x, t)}{\partial t}=-\frac{\partial}{\partial x} h(x, t) P(x, t)+\frac{\partial^{2}}{\partial x^{2}} D(x, t) P(x, t)$,

where $P(x, t) d x$ is the fraction of individuals in the population with reproductive reserves $X_{t} \in[x, x+d x)$ at time $t, h(x, t)$ is commonly called the "drift" factor, and $D(x, t)$ is the "diffusion" factor. Defining $J(x, t)$ as the rate of change of the fraction of individuals in the population with reserve $x$ at time $t$,

$J(x, t)=h(x, t) P(x, t)-\frac{\partial}{\partial x} D(x, t) P(x, t)$,

we can rewrite the Fokker-Planck equation as

$\frac{\partial P(x, t)}{\partial t}=-\frac{\partial}{\partial x} J(x, t)$.

When the reserves $X_{t}$ reach a value 1, spawning occurs and the reserves are reset to 0 . Thus, $X_{t}=1$ is an absorbing state. This condition is enforced by the boundary condition (BC):

BC 1: $\quad P(1, t)=0$.
The rate of spawning is the rate of transitions across the boundary at $X_{t}=1$ :

$$
\begin{aligned}
J(1, t) & =h(1, t) P(1, t)-\left.\frac{\partial}{\partial x} D(x, t) P(x, t)\right|_{x=1} \\
& =-\left.\frac{\partial}{\partial x} D(x, t) P(x, t)\right|_{x=1} .
\end{aligned}
$$

Individuals that spawn reset their reserves to 0 and start allocating energy toward reproduction again. In other words, individuals undergoing transitions across $X_{t}=1$ are reintroduced into the system as individuals with $X_{t}=0$ :

BC 2: $J(1, t)=J(0, t) \Rightarrow h(0, t) P(0, t)$

$$
\begin{aligned}
& -\left.\frac{\partial}{\partial x} D(x, t) P(x, t)\right|_{x=0} \\
= & -\left.\frac{\partial}{\partial x} D(x, t) P(x, t)\right|_{x=1},
\end{aligned}
$$

substituting from Eq. 8. The solution to Eq. 7 is computed by starting with a perfectly coherent collection of individuals, $P(x, 0)=100 \delta(x)$. Equation 7 is solved numerically with boundary conditions $\mathrm{BC} 1$ and $\mathrm{BC} 2$ by discretization using the Crank-Nicholson method (Ascher and Petzold 1998).

We are interested in the asymptotic spawning patterns of the population after the transients have disappeared:

$F^{\infty}(t)=\lim _{t \rightarrow \infty} J(1, t)$.

The above equation achieves convergence with the periodicity of input driver if the spawning patterns are periodic, $F^{\infty}(t+1)=F^{\infty}(t)$. A sufficient condition for the solution $J(1, t)$ to be periodic is that the drift and diffusion factors are periodic

$h(x, t+1)=h(x, t)$ and $D(x, t+1)=D(x, t)$.

Under the above condition, it can be verified that Eq. 7 along with $\mathrm{BC} 1$ and $\mathrm{BC} 2$ are invariant and that $P(x, t)$ and $P(x, t+1)$ are solutions to the same set of equations.

\section{Appendix C: Entrainment under purely time-dependent allocation and maintenance}

Bioenergetic models with only time dependence, i.e., $f\left(X_{t}, t\right)=f(t)$ and $\lambda\left(X_{t}, t\right)=\lambda(t)$, are governed by the following equation derived from Eq. 6 in the absence of noise

$d X_{t}=h(t) d t=\lambda(t) S_{t} d t$, 
where $h(t+1)=h(t)$ and $h(t)>0$. The right-hand side is periodic by our choice of allocations and can therefore be always written as sum of two periodic terms $h_{z}(t)$ and $h_{n z}(t)$ such that

$$
\int_{0}^{1} h_{z}(\tau) d \tau=0 \text { and } \int_{0}^{1} h_{n z}(\tau) d \tau=I, I>0
$$

To achieve a $r: q$ mode-lock, i.e., $q$ firings in $r$ cycles of the input,

$$
\int_{0}^{q} d X_{t}=\int_{0}^{r} h(t) d t \Rightarrow q=r \int_{0}^{1} h_{n z}(t)
$$

utilizing the independence of the allocation strategy on the reserve level $X_{t}$. This is equivalent to a condition on $h_{n z}(t)$ for each allocation strategy. Thus, to achieve a $r: 1$ mode-lock, we get conditions of the form $T_{0}=G\left(r, \alpha, P^{\prime}\right)$, where $P^{\prime}$ represents other model parameters, for each model and allocation strategy. However, the set of parameter values $T_{0}$ satisfying these conditions is, in general, a set of measure zero for a given set of parameter values. In other words, perfect matching of the parameters of the model and the driving signal is necessary for achieving the required entrainment. Therefore, entrainment in such scenarios lacks robustness and the addition of the noise perturbs the system away from these optimal parameter values; we corroborate this fact with simulations in the "Simple reserve accumulator" section.

\section{References}

Ascher UM, Petzold LR (1998) Computer methods for ordinary differential equations and differential-algebraic equations. SIAM, Philadelphia

Atkinson S, Atkinson MJ (1992) Detection of estradiol-17 $\beta$ during a mass coral spawn. Coral Reefs 11(1):33-35. doi:10.1007/ BF00291932

Babcock R, Mundy C, Keesing J, Oliver J (1992) Predictable and unpredictable spawning events - in-situ behavioraldata from free-spawning coral-reef invertebrates. Invertebr Reprod Dev 22:213-228

Babcock RC, Bull GD, Harrison PL, Heyward AJ, Oliver JK, Wallace CC, Willis BL (1986) Synchronous spawnings of 105 scleractinian coral species on the great barrier reef. Mar Biol 90(3):379-394. doi:10.1007/BF00428562

Baird AH, Guest JR, Willis BL (2009) Systematic and biogeographical patterns in the reproductive biology of scleractinian corals. Annu Rev Ecol Evol Syst 40(1):551-571. doi:10.1146/annurev.ecolsys.110308.120220

Bastidas C, Cróquer A, Zubillaga AL, Ramos R, Kortnik V, Weinberger C, Márquez LM (2005) Coral mass- and split-spawning at a coastal and an offshore venezuelan reefs, southern Caribbean. Hydrobiologia 541(1):101-106. doi:10.1007/s10750-004-4672-y
Brady A, Hilton J, Vize P (2009) Coral spawn timing is a direct response to solar light cycles and is not an entrained circadian response. Coral Reefs 28(3):677-680. doi:10.1007/ s00338-009-0498-4

Braley RD (1984) Reproduction in the giant clams Tridacna gigas and T. derasa in situ on the north-central Great Barrier Reef, Australia, and Papua New Guinea. Coral Reefs 3(4):221227. doi:10.1007/BF00288258

Bulsara AR, Elston TC, Doering CR, Lowen SB, Lindenberg K (1996) Cooperative behavior in periodically driven noisy integrate-fire models of neuronal dynamics. Phys Rev, E 53(4):3958. doi:10.1103/PhysRevE.53.3958

Coombes S, Bressloff PC (1999) Mode locking and Arnold tongues in integrate-and-fire neural oscillators. Phys Rev, E 60(2):2086. doi:10.1103/PhysRevE.60.2086

Crean AJ, Marshall DJ (2008) Gamete plasticity in a broadcast spawning marine invertebrate. Proc Natl Acad Sci U S A 105(36):13508-13513. doi:10.1073/pnas.0806590105

Edmunds PJ, Davies PS (1986) An energy budget for Porites porites (Scleractinia). Mar Biol 92(3):339-347. doi:10.1007/ BF00392674

Engelbrecht JR, Mirollo R (2009) Dynamical phase transitions in periodically driven model neurons. Phys Rev, E Stat Nonlin Soft Matter Phys 79(2):021905-1-021905-5. doi:10.1103/ PhysRevE.79.021904

Giese AC (1959) Comparative physiology: annual reproductive cycles of marine invertebrates. Annu Rev Physiol 21(1):547576. doi:10.1146/annurev.ph.21.030159.002555

Giese AC (1966) Lipids in the economy of marine invertebrate. Physiol Rev 46(2):244-298

Gurney W, Crowley P, Nisbet R (1992) Locking life-cycles onto seasons: circle-map models of population dynamics and local adaptation. J Math Biol 30(3):251-279. doi:10.1007/ BF00176151

Katok A, Hasselblatt B (1996) Introduction to the modern theory of dynamical systems. Cambridge Univ. Press, Cambridge

Keener JP, Hoppensteadt FC, Rinzel J (1981) Integrate-and-Fire models of nerve membrane response to oscillatory input. SIAM J Appl Math 41(3):503-517. doi:10.1137/0141042

Knight BW (1972) Dynamics of encoding in a population of neurons. J Gen Physiol 59(6):734-766. doi:10.1085/jgp.59. 6.734

Knowlton N, Maté JL, Guzmán HM, Rowan R, Jara J (1997) Direct evidence for reproductive isolation among the three species of the Montastraea annularis complex in Central America (Panamá and honduras). Mar Biol 127(4):705-711. doi:10.1007/s002270050061

Kooijman S (2000) Dynamic energy and mass budgets in biological systems, 2nd edn. Cambridge Univ. Press, Cambridge

Mangubhai S, Harrison PL (2008) Asynchronous coral spawning patterns on equatorial reefs in Kenya. Mar Ecol Prog Ser 360:85-96. doi:10.3354/meps07385

Mendes JM, Woodley JD (2002) Timing of reproduction in Montastraea annularis: relationship to environmental variables. Mar Ecol Prog Ser 227:241-251

Muller EB, Kooijman SA, Edmunds PJ, Doyle FJ, Nisbet RM (2009) Dynamic energy budgets in syntrophic symbiotic relationships between heterotrophic hosts and photoautotrophic symbionts. J Theor Biol 259(1):44-57. doi: 10.1016/j.jtbi.2009.03.004

Muscatine L, Falkowski PG, Porter JW, Dubinsky J (1984) Fate of photosynthetic fixed carbon in light- and Shade-Adapted colonies of the symbiotic coral Stylophora pistillata. Proc R Soc Lond B Biol Sci 222(1227):181-202. doi:10.1098/rspb. 1984.0058 
Penland L, Kloulechad J, Idip D, Woesik R (2004) Coral spawning in the western Pacific Ocean is related to solar insolation: evidence of multiple spawning events in Palau. Coral Reefs 23(1):133-140. doi:10.1007/s00338-003-0362-x

Plesser H, Tanaka S (1997) Stochastic resonance in a model neuron with reset. Phys Lett A 225:228-234

Rinkevich B (1989) The contribution of photosynthetic products to coral reproduction. Mar Biol 101(2):259-263. doi:10.1007/BF00391465

Risken H (1996) The Fokker-Planck equation : methods of solution and applications, 2nd edn. Springer, Berlin

Satake A, Iwasa Y (2000) Pollen coupling of forest trees: forming synchronized and periodic reproduction out of chaos. J Theor Biol 203(2):63-84. doi:10.1006/jtbi.1999. 1066

Satake A, Iwasa Y (2002) The synchronized and intermittent reproduction of forest trees is mediated by the Moran effect, only in association with pollen coupling. J Ecol 90(5):830838. doi:10.1046/j.1365-2745.2002.00721.x

Starr M, Himmelman JH, Therriault J (1990) Direct coupling of marine invertebrate spawning with phytoplankton blooms. Science 247(4946):1071-1074. doi:10.1126/science. 247.4946.1071
To T, Henson M, Herzog E, Doyle F (2007) A molecular model for intercellular synchronization in the mammalian circadian clock. Biophys J 92(11):3792-3803

van Kampen NG (2007) Stochastic processes in physics and chemistry, 3rd edn. Elsevier, Amsterdam

Vargas-Ángel B, Colley S, Hoke S, Thomas J (2006) The reproductive seasonality and gametogenic cycle of Acropora cervicornis off Broward County, Florida, USA. Coral Reefs 25(1):110-122. doi:10.1007/s00338-005-0070-9

Wallace CC (1985) Reproduction, recruitment and fragmentation in nine sympatric species of the coral genus Acropora. Mar Biol 88(3):217-233. doi:10.1007/BF00392585

Willis B, Babcock R, Harrison P, Oliver J, Wallace C (1985) Patterns in the mass spawning of corals on the great barrier reef from 1981 to 1984. In: Proc int coral reef symp, vol 4, pp 343-348

van Woesik R, Lacharmoise F, Koksal S (2006) Annual cycles of solar insolation predict spawning times of Caribbean corals. Ecol Lett 9(4):390-398. doi:10.1111/j.1461-0248.2006. 00886.x

Yamauchi A (1996) Theory of mast reproduction in plants: storage-size dependent strategy. Evolution 50(5):1795-1807. http://www.jstor.org/stable/2410737 